\title{
Characterization of RsMYB28 and RsMYB29 transcription factor genes in radish (Raphanus sativus L.)
}

X.B. Luo ${ }^{1 *}$, Z. Liu ${ }^{1 *}$, L. Xu' ${ }^{1}$ Y. Wang ${ }^{1}$, X.W. Zhu ${ }^{2}$, W. Zhang ${ }^{1}$, W. Chen ${ }^{1}$, Y.L. Zhu ${ }^{1}$, X.J. Su${ }^{3}$, M. Everlyne ${ }^{1}$ and L.W. Liu ${ }^{1}$

${ }^{1}$ National Key Laboratory of Crop Genetics and Germplasm Enhancement, College of Horticulture, Nanjing Agricultural University, Nanjing, China ${ }^{2}$ Department of Plant Sciences, North Dakota State University, Fargo, ND, USA ${ }^{3}$ Institute of Vegetable Sciences, Jiangsu Academy of Agricultural Sciences, Nanjing, China

*These authors contributed equally to this study.

Corresponding author: L.W. Liu

E-mail: nauliulw@njau.edu.cn

Genet. Mol. Res. 15 (3): gmr.15038381

Received January 4, 2016

Accepted July 14, 2016

Published September 23, 2016

DOI http://dx.doi.org/10.4238/gmr.15038381

Copyright (C) 2016 The Authors. This is an open-access article distributed under the terms of the Creative Commons Attribution ShareAlike (CC BY-SA) 4.0 License

ABSTRACT. Glucosinolates (GSLs) are important secondary metabolites in Brassicaceae plants. Previous studies have mainly focused on GSL contents, types, and biosynthesis-related genes, but the molecular characterization patterns of GSL biosynthesis-related transcription factors remain largely unexplored in radish (Raphanus sativus L.). To isolate transcription factor genes regulating the GSL biosynthesis, genomic DNA and cDNA sequences of RsMYB28 and RsMYB29 genes were isolated in radish. Two R2R3-MYB domains were identified in the deduced amino acid sequences. Subcellular localization and yeast-one hybrid assays indicated that both the 
RsMYB28 and RsMYB29 genes were located in the nucleus and possessed transactivation activity. Reverse transcription quantitative analysis showed that the $R s M Y B 28$ and $R s M Y B 29$ genes were expressed in seeds, leaves, stems, and roots at the seedling, taproot thickening, and mature stages. Both genes were highly expressed during the seedling and taproot thickening stages. The expression level of RsMYB 28 was found to be up-regulated following wounding, glucose, and abscisic acid treatments, whereas RSMYB29 was up-regulated following wounding and methyl jasmonate treatments. These results provide insights into the biological function and characterization of the RsMYB28 and RsMYB29 genes, and facilitate further dissection of the molecular regulatory mechanism underlying the GSL biosynthesis in radish.

Key words: Radish; Glucosinolate; MYB transcription factor; RsMYB28; RsMYB29; RT-qPCR

\section{INTRODUCTION}

Glucosinolates (GSLs) are amino acid-derived natural plant products in the Brassicaceae family, which are well known for their role as a degradation product that induces resistance against generalist herbivores (Kos et al., 2012) and microorganisms (Brader et al., 2006) in plants, as well as for their cancer-preventing properties in humans. In addition, GSLs have gained increasing significance as flavor compounds and potential biopesticides (Mithen et al., 2003).

Recently, many studies have revealed that the GSL biosynthesis is a tripartite pathway consisting of three stages: side-chain elongation, development of the core structure, and secondary modification of the amino acid side chain (Chen et al., 2011; Augustine et al., 2013a). The most prominent GSL biosynthetic precursor amino acids include methionine, tryptophan, and phenylalanine, which result in aliphatic, indolic, and aromatic GSLs, respectively (Kos et al., 2012).

In recent years, a great number of functional genes involved in the GSL biosynthetic pathway have been successfully identified in several plant species including Arabidopsis thaliana (Sønderby et al., 2010), Brassica juncea (Augustine et al., 2013a), and B. rapa (Kim et al., 2013). The R2R3-MYB proteins are plant specific and constitute one of the largest transcription factor families in plants (Wilkins et al., 2009). Many R2R3-MYB genes have been found to play important roles in a variety of biological functions, including biosynthesis of secondary metabolites (Gigolashvili et al., 2008; Verdier et al., 2012), regulation of biotic and abiotic stressors by increasing hypersensitivity to abscisic acid (ABA) (Shan et al., 2012), precise establishment of the root epidermal pattern, and control of cell cycle progression (Kang et al., 2009; Xie et al., 2010). In Arabidopsis, the AtMYB28, AtMYB29, and AtMYB76 genes positively regulate aliphatic GSL biosynthesis (Gigolashvili et al., 2008; Sønderby et al., 2010; Li et al., 2013), whereas the AtMYB34, AtMYB51, and AtMYB122 genes have been shown to regulate indolic GSL biosynthesis (Gigolashvili et al., 2007a). In $B$. juncea, four homologous genes encoding MYB28 were found to participate in regulating the aliphatic GSL biosynthesis (Augustine et al., 2013a). Moreover, both the MYB28 and MYB29 genes showed high expression levels in flowers, seeds, and stems of Chinese cabbage (Kim et al., 2013). AtMYB28 transcripts were nearly absent, whereas AtMYB29 and AtMYB76 transcripts

Genetics and Molecular Research 15 (3): gmr.15038381 
showed no significant decrease at aliphatic GSL biosynthesis induction in knockout mutants (Gigolashvili et al., 2009; Sønderby et al., 2010). This indicates that AtMYB28 is an important regulator of aliphatic GSL biosynthesis, whereas AtMYB29 and AtMYB76 may play minor roles (Gigolashvili et al., 2009; Sønderby et al., 2010). These findings have provided useful information for the characterization of the MYB28 and MYB29 genes in other vegetable crops.

Radish (Raphanus sativus L., $2 \mathrm{n}=2 \mathrm{x}=18$ ), belonging to the Brassicaceae family, is an important root vegetable crop worldwide. It contains large amounts of anti-oxidants and GSLs (Malik et al., 2010). Three candidate genes, RsMAM3, RsIPMDH1, and RsBCAT4 have been reported as being involved in the 4-methylthio-3-butenyl GSL biosynthesis in radish roots, based on a QTL region synteny analysis of $A$. thaliana and $B$. rapa genome sequences (Zou et al., 2013). With de novo transcriptome sequencing, eight genes were found to be involved in the GSL metabolism-related pathways in radish (Wang et al., 2013). Two cDNA clones of myrosinase, a hydrolyzing GSL enzyme, were isolated and characterized in radish (Pan et al., 2014). However, the MYB transcription factors involved in regulating the GSL biosynthesis remain largely unexplored in radish.

In this study, two R2R3-MYB transcription factors, RsMYB28 and RsMYB29, were isolated from radish and their expression patterns under different treatments and in different tissues at three developmental stages were investigated. Moreover, subcellular localization and transactivation activity analyses of these two transcription factors were performed. The results of this study provide valuable information for functional validation of the RsMYB28 and $R S M Y B 29$ genes, and facilitate further dissecting of the molecular regulatory mechanism of the GSL biosynthesis in root vegetable crops.

\section{MATERIAL AND METHODS}

\section{Plant materials}

The advanced inbred radish line 'NAU-ZQH' was grown in a growth chamber at $25^{\circ} \mathrm{C} / 14$-h light and $18^{\circ} \mathrm{C} / 10$-h dark. At the four-true leaf stage, a wounding treatment was adopted by crushing $90 \%$ of the area of a leaf using a hemostat (Jun et al., 2011; Miao et al., 2013). The leaves were treated with methyl jasmonate (MeJA, $100 \mu \mathrm{M})$, ABA $(100 \mu \mathrm{M})$, glucose $(100 \mu \mathrm{M})$, and sorbitol (as an osmotic control) $(100 \mu \mathrm{M})$ (Jun et al., 2011). Three individuals were used for each treatment, and three replicates of leaves for each treatment were collected at different time points $(0,1,2,4,6,8,12$, and $24 \mathrm{~h})$. For the spatial-temporal expression in different tissues, roots, leaves, and stems were sampled at the seedling, taproot thickening, and mature stages, respectively. Three replicates were obtained for each tissue. At the mature stage, samples of flesh and skin were separated. All samples were frozen immediately in liquid nitrogen and stored at $-80^{\circ} \mathrm{C}$ until further use.

\section{Isolation of cDNA and genomic DNA sequences}

Genomic DNA was isolated from radish root using a modified CTAB method (Liu et al., 2003). Total RNA was isolated from radish samples using a Simple P total RNA extraction kit (Tiangen Biotech Co., Ltd., Beijing, China), following the manufacturer instructions. First-strand cDNA was synthesized with M-MLV (RNase H-) (Takara, Dalian, China) and oligo-dT primers. Based on previously published radish transcriptome sequences (Wang et al.,

Genetics and Molecular Research 15 (3): gmr.15038381 
2013), two gene-specific primer pairs (RsMYB28F1/R1 and $R s M Y B 29 \mathrm{~F} 1 / \mathrm{R} 1)$ were designed to amplify the open reading frame (ORF) of RsMYB28 and RsMYB29, respectively. The polymerase chain reaction (PCR) contained $20 \mathrm{ng}$ genomic DNA or cDNA, $0.2 \mathrm{mM}$ dNTPs, $2.0 \mathrm{mM} \mathrm{MgCl}, 0.8 \mathrm{U}$ Taq DNA polymerase, and $0.4 \mu \mathrm{M}$ each primer. The PCR products were purified and cloned into the pMD18-T vector (Takara). The sequence analysis was performed on an ABI PRISM 3730 Genetic Analyzer (Applied Biosystems, Forster City, CA, USA).

\section{Phylogenetic analysis}

The amino acids of the RsMYB28 and RsMYB29 genes were predicted using the DNASTAR software (Lasergene). A BLASTx of the coding sequences was performed on NCBI (http://blast.ncbi.nlm.nih.gov/Blast.cgi). The retrieved protein sequences that were used for the analysis are listed in Table S1. Sequence alignment was determined by ClustalW (http://align.genome.jp/). The neighbor-joining method, including bootstrap analyses with 1000 replicates, was used to construct a phylogenetic tree using MEGA6 (Tamura et al., 2007).

\section{Subcellular localization of RsMYB28 and RsMYB29}

To generate $p J I T 166-R s M Y B 28(29)-G F P$ (green fluorescent protein) containing an RsMYB28(29)-GFP fusion plasmid under the control of cauliflower mosaic virus $35 \mathrm{~S}$ (CaMV $35 \mathrm{~S})$ promoter, the coding sequences of RsMYB28 and RsMYB29 were cloned into pJIT166GFP vectors with $B a m \mathrm{HI} / X b a \mathrm{I}$ and HindIII/XbaI, respectively. After sequence confirmation, the recombinant plasmids were transformed into onion (Allium cepa) epidermal cells using a gene gun (PDS 1000, Bio-Rad, Hercules, CA, USA) (Yang et al., 2012). The RsMYB28GFP and RSMYB29-GFP fusion proteins in the transformed onion cells were cultured on Murashige and Skoog medium for $24 \mathrm{~h}$ in darkness and observed under a confocal laser scanning microscope (Leica SP2, Leica, Wetzlar, Germany). The images were obtained by automatic recording.

\section{Transactivation activity analysis of $R s M Y B 28$ and $R s M Y B 29$}

The ORF of RsMYB28 and RsMYB29 were inserted into the NcoI/SmaI and EcoRI/ $B a m H I$ cloning sites of the yeast expression vector pGBKT7 to produce $p B D-R s M Y B 28$ and $p B D-R s M Y B 29$, respectively. Both the controls (positive control: pCL1 and negative control: pGBKT7) and the recombinant plasmids were transformed into Saccharomyces cerevisiae strain Y2HGold (Clontech, Palo Alto, CA, USA) (Gao et al., 2012). pCL1 transformants were selected on SD/-Leu medium, while the other transformed yeast strains were plated on SD/Trp medium. After 3 days, all transformed cell lines were streaked onto SD/-His-Ade and $\mathrm{SD} / \mathrm{X}-\alpha$-gal, to investigate the growth response.

\section{Quantitative real-time PCR (RT-qPCR)}

Differences in expression patterns of RsMYB28 and RsMYB29 were analyzed in various tissues from different radish developmental stages. In addition, the different leaf treatments were analyzed using the SYBR Green Master ROX (Roche, Basel, Switzerland). The amplification reactions were incubated at $95^{\circ} \mathrm{C}$ for $30 \mathrm{~s}$, followed by 40 cycles of $95^{\circ} \mathrm{C}$

Genetics and Molecular Research 15 (3): gmr.15038381 
for $5 \mathrm{~s}, 58^{\circ} \mathrm{C}$ for $15 \mathrm{~s}$, and $72^{\circ} \mathrm{C}$ for $20 \mathrm{~s}$. The Actin gene was used as internal control (Xu et al., 2012). Relative gene expression levels were calculated using the $2^{-\Delta \Delta C t}$ method (Livak and Schmittgen, 2001). The primers for the selected transcripts are shown in Table 1.

Table 1. Primer sequences used in this study.

\begin{tabular}{l|l|l}
\hline Primer name & Sequence $\left(5^{\prime}-3^{\prime}\right)$ & Purpose \\
\hline RsMYB28F1 & GGGACCATCACACAATTCATTTCTC & Genomic DNA and cDNA isolation \\
\hline RsMYB28R1 & TTTCTTACTTCTTGCGGTGTCTTA & Genomic DNA and cDNA isolation \\
\hline RsMYB29F1 & GAAGAAGAAGCAAAAAAATGTCAAG & Genomic DNA and cDNA isolation \\
\hline RsMYB29R1 & AACCACGAATTTAACCAGTAAGCAA & Genomic DNA and cDNA isolation \\
\hline RsMYB28F2 & CGCAAGCTTATGTCAAGAAAACCAT & Subcellular localization \\
\hline RsMYB28R2 & TGCTCTAGATATGATTTGCTTCTCG & Subcellular localization \\
\hline RsMYB29F2 & CCCAAGCTTATGTCAAGAAAGGCAT & Subcellular localization \\
\hline RsMYB29R2 & TGCTCTAGAATCTTGGTTCGTATCA & Subcellular localization \\
\hline RsMYB28F3 & CATCCATGGCGAAGAAAATGTCAAG & Transcriptional activation ability \\
\hline RsMYB28R3 & TCCCCCGGGCTCGTTCAGGAAAGTT & Transcriptional activation ability \\
\hline RsMYB29F3 & CCGGAATTCATGTCAAGAAAGGCAT & Transcriptional activation ability \\
\hline RsMYB29R3 & CGCGGATCCCTAATCTTGGTTCGTA & Transcriptional activation ability \\
\hline RsMYB28F4 & CTCTTACATCCACGAACA & RT-qPCR \\
\hline RsMYB28R4 & CTCCTCCGAACTAAACTC & RT-qPCR \\
\hline RsMYB29F4 & GGACCATTGAAGAAGAC & RT-qPCR \\
\hline RsMYB29R4 & CAAGTAGTTAGCCCATCG & RT-qPCR \\
\hline Actin2/7F & GCATCACACTTTCTACAAC & RT-qPCR \\
\hline Actin2/7R & CCTGGATAGCAACATACAT & RT-qPCR \\
\hline
\end{tabular}

\section{RESULTS}

\section{Cloning and characterization of RsMYB28 and RsMYB29 genes}

Using the specific primer pairs $R s M Y B 28 \mathrm{~F} 1 / \mathrm{R} 1$ and $R s M Y B 29 \mathrm{~F} 1 / \mathrm{R} 1$, the genomic DNA and cDNA sequences of RsMYB28/RsMYB29 genes were isolated from radish, respectively. The genomic DNA of RsMYB28 (Figure 1A) contained three exons and two introns spanning $1836 \mathrm{bp}$. The RsMYB28 cDNA clone was $1614 \mathrm{bp}$ in length and consisted of a 354-bp 5'-untranslated region (UTR), 168-bp 3'-UTR, and an ORF of 1092 bp. The ORF encoded 363 amino acids with a calculated molecular mass of $40.8 \mathrm{kDa}$ and a predicted $\mathrm{pI}$ of 5.64 (Figure 1B). The genomic DNA of RsMYB29 spanned $1493 \mathrm{bp}$, with three exons and two introns (Figure 1A). The full-length RsMYB29 cDNA was $1093 \mathrm{bp}$, which was flanked by a 17-bp 5'-UTR and a 53-bp 3'-UTR. RsMYB29 contained a 1023-bp ORF encoding 340 amino acids with a calculated molecular mass of $38.2 \mathrm{kDa}$ and a pI of 4.67 (Figure 1C).

The amino acid sequence alignment indicated that RsMYB28 and RsMYB29 had similar sequence identity with AtMYB28 and AtMYB29, respectively. Our results indicated that RsMYB28 and RsMYB29 have two imperfect sequence repeats (R2: $\mathrm{W}-\mathrm{X}_{19}-\mathrm{W}-\mathrm{X}_{19}-\mathrm{W}$ and R3: $\left.\mathrm{F}-\mathrm{X}_{18}-\mathrm{W}-\mathrm{X}_{18}-\mathrm{W}\right)$ at the N-terminal region, whereas there was a low level of sequence conservation at the C-terminal region. RsMYB28 and RsMYB29 also had a nuclear localization signal sequence, LKKRL, which is also present in the AtMYB28 and AtMYB76 sequences, respectively, followed by an R3 domain (amino acid residues 112-116) (Figure 2).

The phylogenetic tree was constructed based on RsMYB28, RsMYB29, and 18 MYBs from other plant species (Figure 3 ). The tree indicated the formation of three different groups; $R s M Y B 28$ and $R s M Y B 29$ were located in the first and second groups, respectively. RsMYB28 was grouped most closely with Bju_MYB28-1, Bo_MYB28-1, and Bra_MYB28-3, whereas RsMYB29 grouped with Bju_MYB29-2. Thus, they showed close evolutionary relationships with MYB28 and MYB29-like sequences from Brassica species, respectively. 


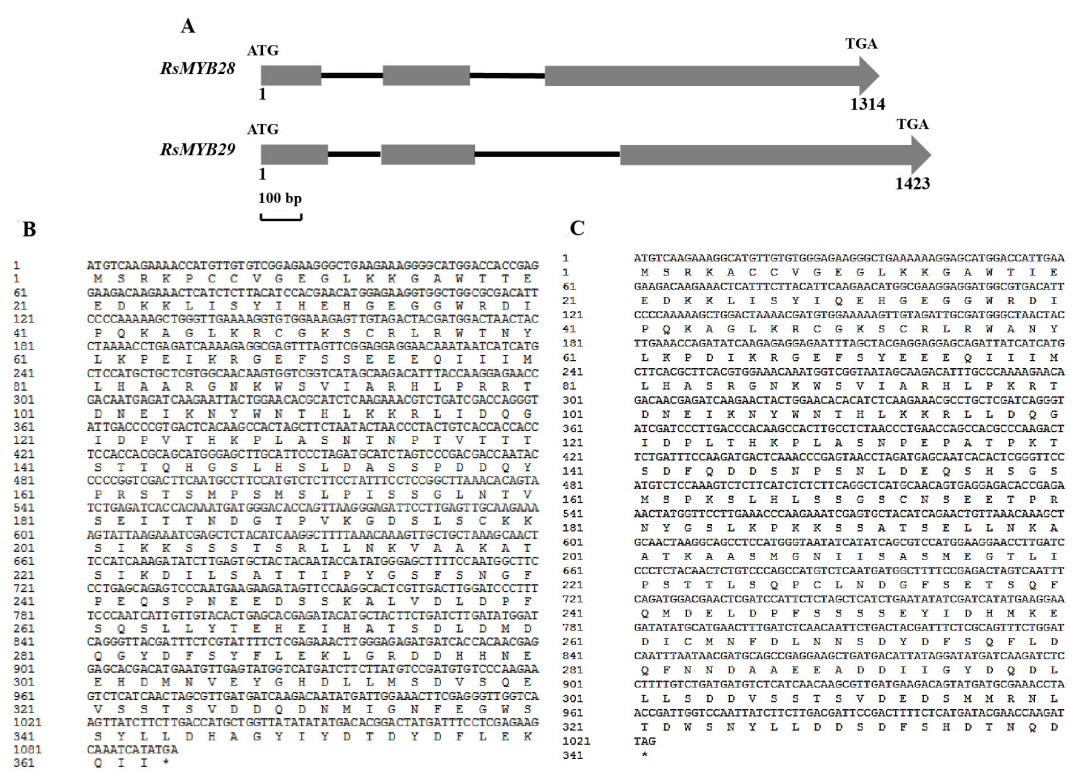

Figure 1. DNA structure and deduced amino acid sequences of RsMYB28 and RsMYB29. A. DNA structure of RsMYB28 and RsMYB29. Exons are highlighted in gray boxes and introns by black lines, the start and stop codons are shown for the entire open reading frame. Nucleotide and deduced amino acid sequences of the RsMYB28 (B) and $R s M Y B 29(\mathbf{C})$.

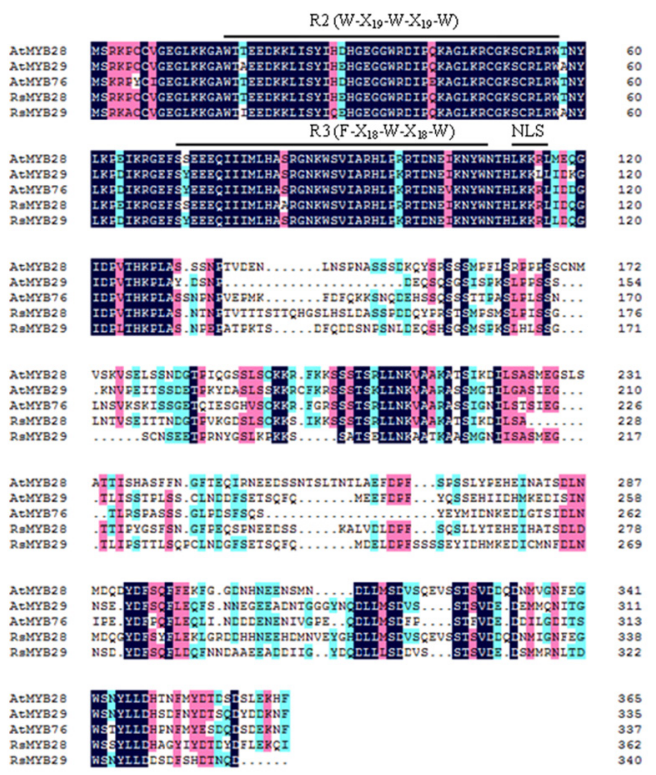

Figure 2. Deduced peptide sequences of RsMYB28 and RsMYB29 and three aliphatic glucosinolate-regulating Arabidopsis thaliana MYB proteins: AtMYB28, AtMYB29, and AtMYB76. Their GenBank accession Nos. are NP_196386, NP_200950, and NP_196387, respectively. Features of the sequence include R2 and R3 domains and a putative nuclear localization signal (NLS, LKKRL), which are indicated by solid lines. Purple, blue and pink background represents amino acid identity of 100,75 and $50 \%$, respectively.

Genetics and Molecular Research 15 (3): gmr.15038381 


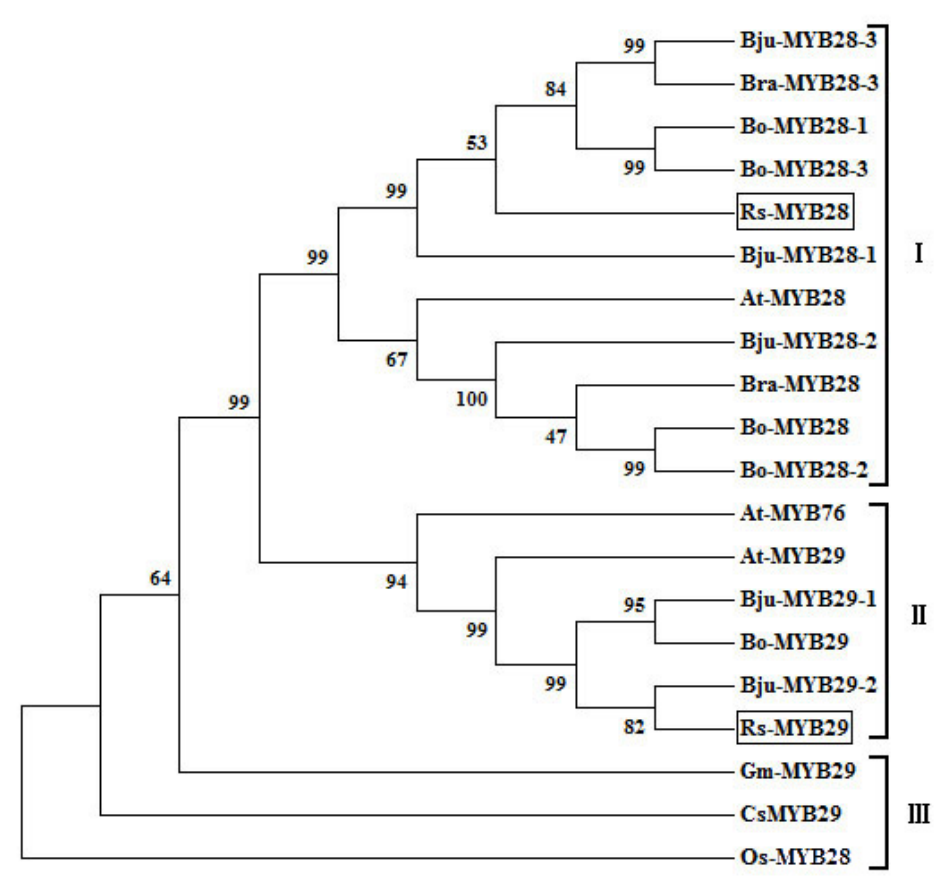

Figure 3. Phylogenetic tree based on RsMYB28, RsMYB29, and homologous sequences from different plant species. Information about which proteins are included in the tree is presented in Table S1. The percentage of replicate trees in which the associated proteins clustered together in the bootstrap test (1000 replicates) is shown next to the branches.

\section{Subcellular localization of RsMYB28 and RsMYB29}

Using predict protein software (http://www.predictprotein.org/), we found that the RsMYB28 and RsMYB29 proteins had a conserved SV40-type putative nuclear localization motif, LKKRL, which is a nucleus-localized protein. To examine the subcellular localization of RsMYB28 and RsMYB29 in living cells, the recombinant constructs of Pro35S:RsMYB28GFP and Pro35S:RsMYB29-GFP fusion genes were introduced into onion epidermal cells by particle bombardment. As compared with the GFP-positive control vector (Figure 4A), both RsMYB28-GFP and RsMYB29-GFP transiently transformed cells showed a strong green fluorescence signal in the nucleus (Figure 4B and C), implying that they might be localized in the nucleus.

\section{Transcriptional activation of $R s M Y B 28$ and $R s M Y B 29$}

To investigate the transcriptional activity of RsMYB28 and $R s M Y B 29$, they were fused into the pGBKT7 vector (Figure 5A and B) and then transformed into the yeast strain Y2HGold (Clontech). As shown in Figure $5 \mathrm{C}$ and D, the yeast cells co-transformed with pCL1 (positive control), $p G B K T 7-R s M Y B 28$, and $p G B K T 7-R s M Y B 29$ grew normally in SD/-HisAde medium and $\mathrm{SD} / \mathrm{X}$ - $\alpha$-gal medium with a clear blue polyline (Figure $5 \mathrm{D}$ and $\mathrm{E}$ ). In contrast,

Genetics and Molecular Research 15 (3): gmr.15038381 
the co-transformed yeast cells containing pGBKT7 (the negative control) failed to grow on both $\mathrm{SD} /$-His-Ade and $\mathrm{SD} / \mathrm{X}-\alpha$-gal media. These results indicated that both the RsMYB28 and $R s M Y B 29$ genes have transcriptional activity in the yeast cell. This transcriptional activity may be indispensable for the roles that these genes play in regulating gene expression in radish.
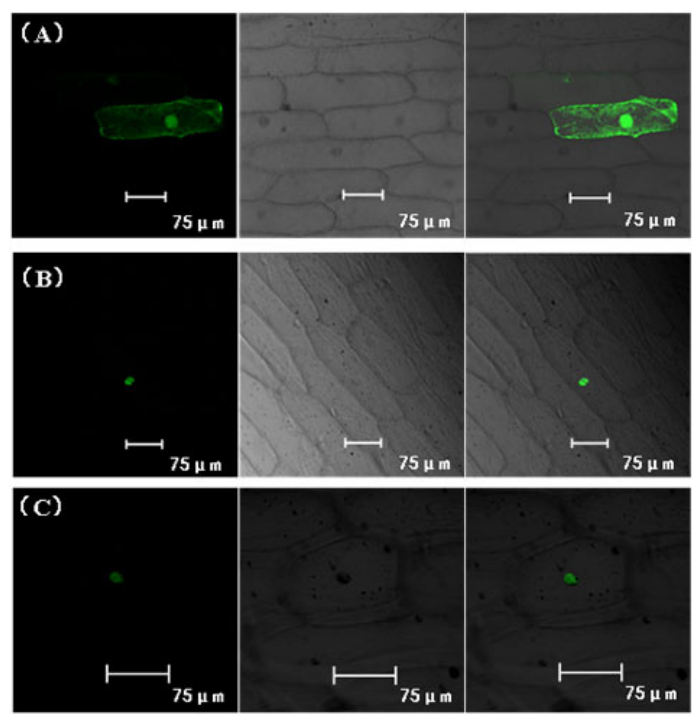

Figure 4. Subcellular localization of RsMYB28 and RsMYB29 proteins in onion (Allium cepa) epidermis cells. Onion epidermis cells transformed with GF-positive control vector (A), Pro35S:RsMYB28-GFP (B), and Pro35S:RsMYB29-GFP (C).

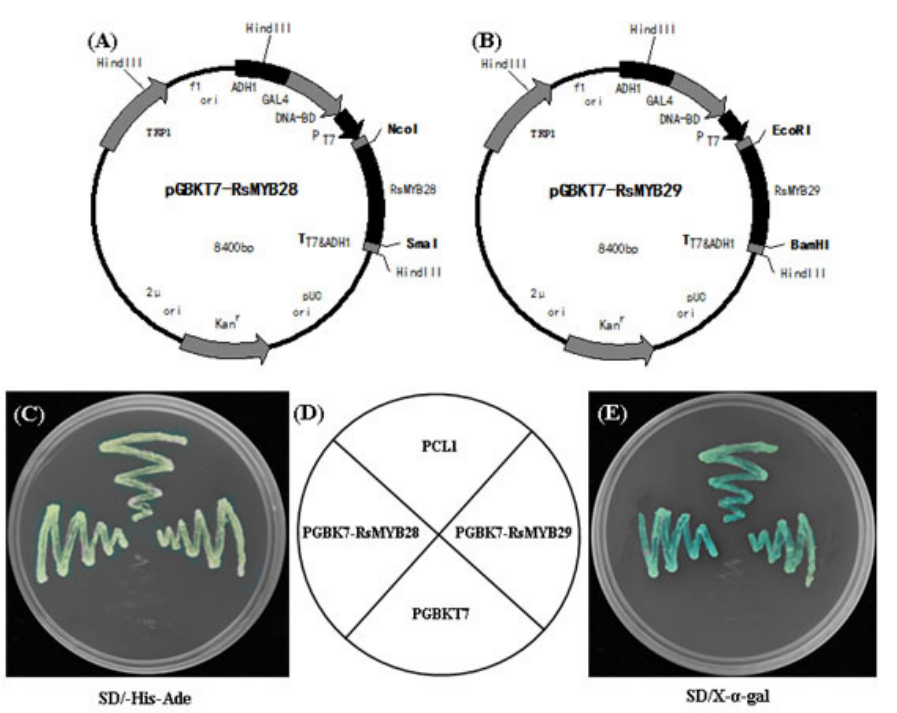

Figure 5. Structure of the $R S M Y B 28$ (A) and $R S M Y B 29$ (B) vectors; growth of transformed yeast cells grown on SD without histidine and adenine $(\mathbf{C})$; position of each yeast strain $(\mathbf{D})$; and X- $\alpha$-gal activity $(\mathbf{E})$. 


\section{Expression analysis of $R s M Y B 28$ and $R s M Y B 29$ in response to different treatments}

RT-qPCR was carried out to analyze the dynamic expression patterns of RsMYB28 and RsMYB29. The differential expression patterns of RsMYB28 and RsMYB29 in different organs at three main developmental stages, compared with the constitutively expressed Actin gene, are shown in Figure 6. RsMYB28 and RsMYB29 were expressed in most of the tissue types and showed similar expression patterns. In the stem, the expression of RsMYB28 and RSMYB29 at the taproot thickening stages was 13.6- and 97-fold higher than that at the mature stage, respectively.

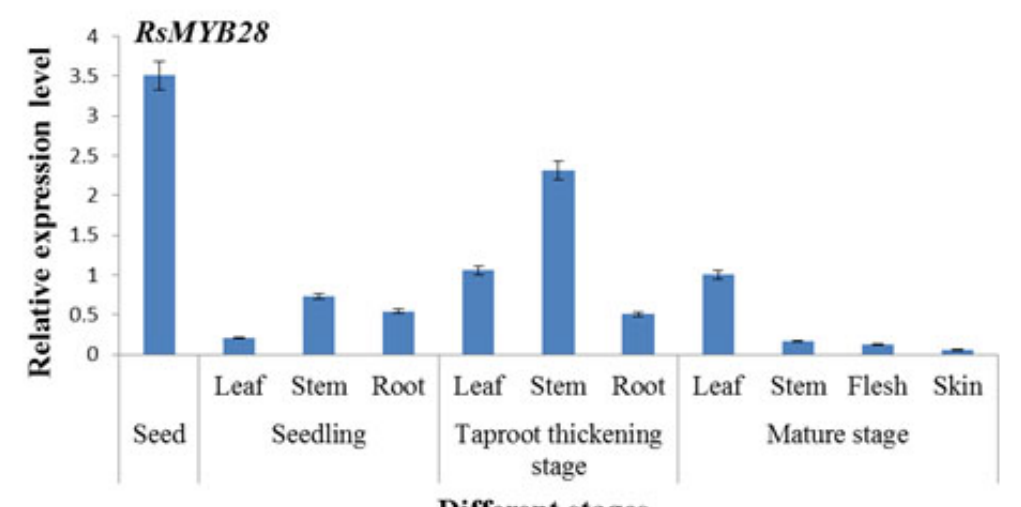

\section{Different stages}

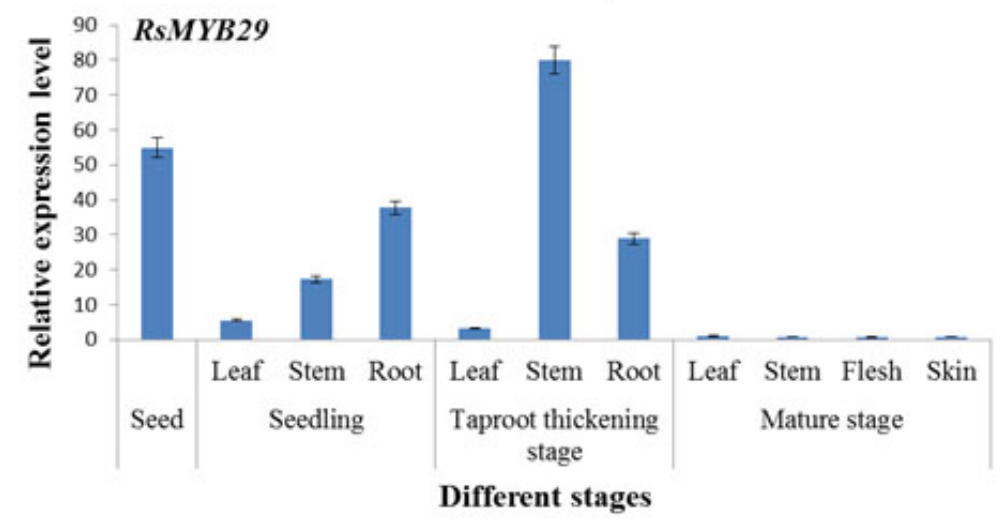

Figure 6. Expression levels of RsMYB28 and RsMYB29 genes in different tissues at different developmental stages. Actin 2/7 was used as a constitutively expressed Actin gene. Independent data were analyzed in triplicate, and the error bars represent standard deviations.

The expression of RsMYB28 and RsMYB29 under the different treatments wounding, MeJA, ABA, and glucose were profiled using RT-qPCR (Figure 7). In the wounding treatment, the expression level of the $R S M Y B 28$ gene showed a gradual increase until $24 \mathrm{~h}$, whereas the RsMYB29 gene dramatically increased at $4 \mathrm{~h}$ followed by a strong decrease at $6 \mathrm{~h}$. In the MeJA treatment, the expression level of RsMYB29 steadily increased with a peak at $8 \mathrm{~h}$, followed by a steady decrease. In contrast, RsMYB28 expression remained constant. Under 
ABA stress, the $R s M Y B 28$ gene showed a dramatic rise in expression level with a peak at 8 $\mathrm{h}$, followed by a decrease at $10 \mathrm{~h}$. RsMYB29 expression was not markedly affected by ABA stress. In the case of the exogenous glucose treatment (using sorbitol as osmotic control), the expression level of RsMYB28 dramatically increased after $4 \mathrm{~h}$, with a peak at $8 \mathrm{~h}$, followed by a decrease. In contrast, $R s M Y B 29$ only showed a slight increase at $6 \mathrm{~h}$. Thus, the expression of RsMYB28 appeared to be induced in the wounding, glucose, and ABA treatments, whereas $R s M Y B 29$ expression was triggered in the wounding and MeJA treatments. This indicates that the regulatory role of RsMYB28 may be greater than that of RsMYB29 under some treatments.

A
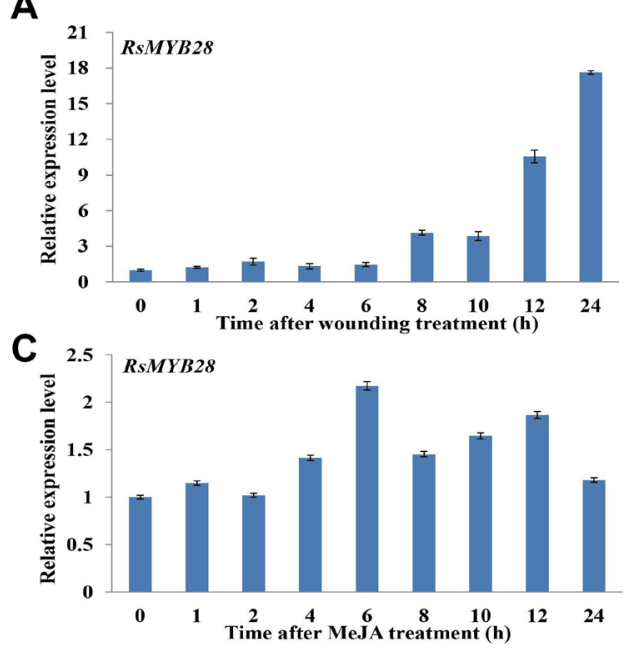

$\mathrm{E}$

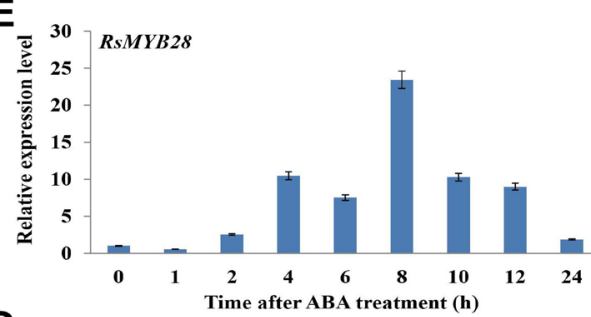

G

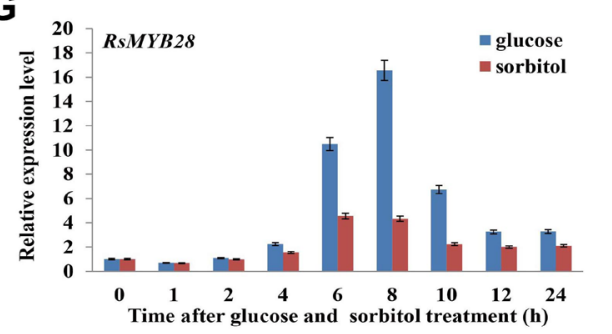

B

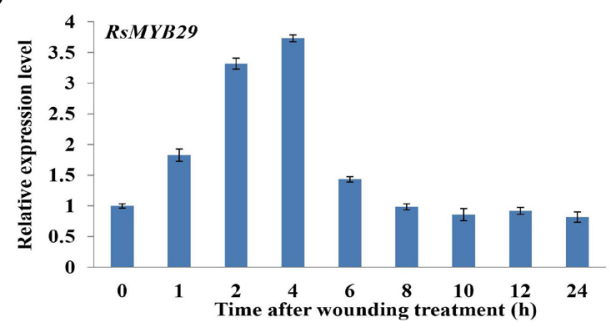

D $\quad 0.24]_{R S M Y B 29}$

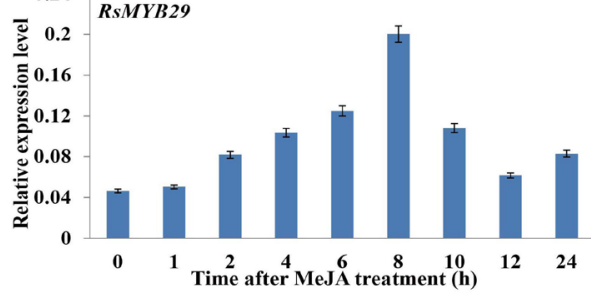

$\mathbf{F}$

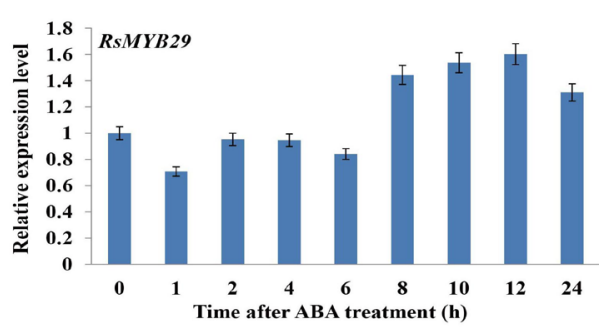

H

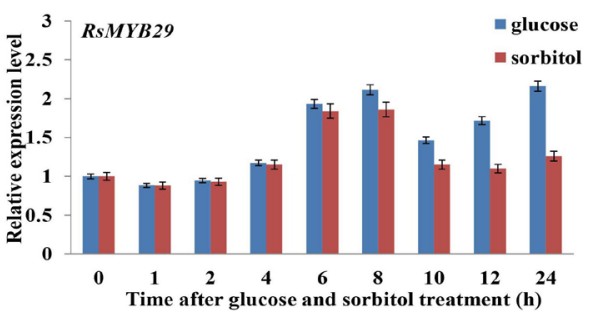

Figure 7. Expression patterns of RsMYB28 and RsMYB29 subjected to various treatments in radish, as assayed by RT-qPCR. A. and B. Wounding treatment, C. and D. treated with $100 \mu \mathrm{M}$ methyl jasmonate (MeJA), E. and F. treated with $100 \mu \mathrm{M}$ abscisic acid (ABA), and G. and H. treated with $100 \mu \mathrm{M}$ glucose. Actin2/7 was used as a constitutively expressed Actin gene. Independent data were analyzed in triplicate and the error bars represent the standard deviations.

Genetics and Molecular Research 15 (3): gmr.15038381 


\section{DISCUSSION}

\section{Genomic DNA and phylogeny of RsMYB28 and RsMYB29 genes}

Two R2R3-MYB transcriptional factors, MYB28 and MYB29, along with MYB76, have been identified as regulators of aliphatic GSL biosynthesis in A. thaliana, B. juncea, and B. rapa (Hirai et al., 2007; Gigolashvili et al., 2007b, 2008; Li et al., 2013). However, there has been no study on the MYB28 and MYB29 coding sequences in radish. In this study, the genomic DNA and cDNA of RsMYB28 and RsMYB29 were isolated. Like the BjuMYB28 gene of other R2R3-MYB members (Augustine et al., 2013a), both genes were found to be conserved with three separated exons and two introns (Figure 1). The amino acid sequence alignment showed that both RsMYB28 and RsMYB29 contained two highly conserved domains, and showed high homology with AtMYB28 and AtMYB29, respectively. This indicates a potential similarity in function (Figure 2). In contrast, the RsMYB28 and RsMYB29 proteins showed structural variation compared with the AtMYB28, AtMYB29, and AtMYB76 proteins in C-terminal region, which will lead to differential gene functions in the regulation of the aliphatic GSL biosynthesis.

Our phylogenetic analysis showed that $R s M Y B 28$ clustered together with $M Y B 28$ from different Brassicaceae species, whereas AtMYB76 clustered with RsMYB29 (Figure 3). This is in line with what has previously been reported (Araki et al., 2013). A previous comparison of the $R$. sativus genome structure with that of the A, B, and C genomes of Brassica species revealed extensive chromosome homology among Brassicaceae species (Li et al., 2011). In our study, the RsMYB28 and RsMYB29 proteins were grouped with the Bju_MYB28s and Bju_MYB29s proteins, respectively, indicating that RsMYB28 and RsMYB $\overline{2} 9$ have a high level of sequence conservation with their homologues from the $B$. juncea genomes. Silencing of the BjMYB28 transcription factor gene caused a reduction in the GSL content in B. juncea (Augustine et al., 2013b). BoMYB29 has been shown to be a regulator for increasing methylsulfinyl GSL content in B. oleracea (Araki et al., 2013). Therefore, these two genes may have similar biological functions in Brassicaceae species in the aliphatic GSL biosynthesis.

\section{Subcellular localization and transactivation analysis of $R s M Y B 28$ and $R S M Y B 29$}

The subcellular localization of MYB28 in A. thaliana and B. juncea was found to be in the nucleus (Gigolashvili et al., 2007b; Augustine et al., 2013a). Our GFP transient expression indicated that RsMYB28 and RsMYB29 were both located in the nuclei in onion epidermal cells and also possessed transcriptional activity. This may be indicative of an expressionregulating function in the nucleus (Figures 4 and 5). Previous studies have indicated that AtMYB28 is an important regulator and AtMYB29 only plays a minor role, whereas AtMYB76 has an accessory role (Gigolashvili et al., 2009; Sønderby et al., 2010).

\section{Expression levels of $R s M Y B 28$ and $R s M Y B 29$ at different stages and in different organs}

MYB28 and MYB29 showed high expression levels in the flowers, seeds, and stems, but the expression was dramatically different in the stem when compared with that in other Chinese cabbage organs (Kim et al., 2013). In our study, the expression of RsMYB28 and RsMYB29 could be detected in all examined tissues including seeds, roots, stems, and leaves,

Genetics and Molecular Research 15 (3): gmr.15038381 
especially in seeds and stems (Figure 6), which is in agreement with a previous study reporting that RsMYB28 and RsMYB29 exhibited high expression level in the stem, and seed (Kim et al., 2013). Augustine et al. (2013a) reported that the expression levels of four BjuMYB28 homologues were higher in seedlings, stems, and siliques than in roots and primary leaf tissues in $B$. juncea. In addition, an expression analysis using RNA-seq in sugar beet revealed that the highest number of $B v M Y B$ genes was expressed in inflorescences, followed by seedlings, taproots, seeds, and young leaves (Stracke et al., 2014). In the present study, both RsMYB28 and $R S M Y B 29$ exhibited high expression levels at the seedling and taproot thickening stage. Therefore, it could be suggested that RsMYB28 and RsMYB29 may play crucial roles in radish growth.

\section{Expression patterns of $R s M Y B 28$ and $R s M Y B 29$ under various treatments}

GSLs are important secondary metabolites for plant defense (Grubb and Abel, 2006). Various environmental stimuli such as wounding, herbivore attacks, MeJA, salicylic acid (SA), and 1-aminocyclopropane (ACC) have been reported to influence GSL metabolism (Mikkelsen et al., 2003). Interestingly, several GSL biosynthetic regulatory genes were found to be up-regulated by MeJA, SA, and ACC (Gigolashvili et al., 2007b, 2009; Sønderby et al., 2010). In A. thaliana, MYB28 and MYB29 play a vital role in aliphatic GSL biosynthesis (Gigolashvili et al., 2009). Our results showed that the expression level of RsMYB28 increased quickly in response to wounding. RsMYB29 showed a transient induction following wounding, as was also shown in MYB28, MYB29, and the aliphatic GSL biosynthetic genes BCAT4 and MAM1 in A. thaliana (Schuster et al., 2006). The spatial expression patterns of the RsMYB28 and $R s M Y B 29$ genes suggested that they might play a synergistic action in resistance against herbivores (Figure 7A and B). The expression of RsMYB29 was up-regulated by MeJA treatment, whereas that of RsMYB28 remained unaffected (Figure 7C and D). This result is in accordance with the MYB28 and MYB29 expression patterns found for aliphatic GSL synthesis regulation in Chinese cabbage after MeJA treatment (Zang et al., 2015).

There is little known about MYB28 and MYB29 induction after ABA treatment. Under drought, salt, or cold conditions, $\mathrm{ABA}$ is often regarded as the primary signal for plant resistance against abiotic stress (Shinozaki and Yamaguchi-Shinozaki, 2007). In this study, RsMYB28 was significantly induced by ABA treatment, whereas RsMYB29 was not affected (Figure 7E and F), implying that $R S M Y B 28$ may be involved in plant defense against abiotic stress.

In addition, compared with sorbitol, glucose can greatly induce aliphatic GSLs through MYB transcription factors (Gigolashvili et al., 2007b; Miao et al., 2013). In this study, the expression level of RsMYB29 was lower than that of $R S M Y B 28$, suggesting a predominating role of $R S M Y B 28$ for regulation of aliphatic GSL biosynthesis by glucose induction (Figure $7 \mathrm{G}$ and $\mathrm{H}$ ). Furthermore, the expression of RsMYB28 showed a 16.6-fold increase after $8 \mathrm{~h}$ of glucose treatment, which is consistent with previous findings in $A$. thaliana subjected to glucose treatment (Gigolashvili et al., 2007b).

In the present study, genomic DNA and cDNA of RsMYB28 and RsMYB29 genes were isolated and characterized in radish. The RSMYB28 and $R S M Y B 29$ were found to be localized in the nucleus and possessed transactivation activity. Both the RsMYB28 and RsMYB29 genes were strongly expressed at the seedling and taproot thickening stages, and their expression patterns were up-regulated after wounding. The RsMYB28 gene was induced by ABA and glucose, whereas RsMYB29 was induced only by MeJA. It could be concluded that the 
RsMYB28 and RsMYB29 genes play a synergistic role in the resistance against biotic and abiotic stressors through different signaling pathways. These findings provide useful information for functional analysis of the R2R3-MYB transcription factor family, and facilitate the dissection of the molecular regulatory mechanisms underlying the GSL biosynthesis in radish.

\section{Conflicts of interest}

The authors declare no conflict of interest.

\section{ACKNOWLEDGMENTS}

Research in part supported by grants from the National Key Technology Research and Development Program of China (\#2016YFD0100204-25), the Jiangsu Agricultural Science and Technology Innovation Fund [\#CX(16)1012], and the Key Technology R\&D Program of Jiangsu Province (\#BE2016379, \#BE2013429).

\section{REFERENCES}

Araki R, Hasum IA, Nishizawa OI, Sasaki K, et al. (2013). Novel bioresources for studies of Brassica oleracea: identification of a kale MYB transcription factor responsible for glucosinolate production. Plant Biotechnol. J. 11: 1017-1027. http://dx.doi.org/10.1111/pbi.12095

Augustine R, Majee M, Gershenzon J and Bisht NC (2013a). Four genes encoding MYB28, a major transcriptional regulator of the aliphatic glucosinolate pathway, are differentially expressed in the allopolyploid Brassica juncea. $J$. Exp. Bot. 64: 4907-4921. http://dx.doi.org/10.1093/jxb/ert280

Augustine R, Mukhopadhyay A and Bisht NC (2013b). Targeted silencing of BjMYB28 transcription factor gene directs development of low glucosinolate lines in oilseed Brassica juncea. Plant Biotechnol. J. 11: 855-866. http://dx.doi. org/10.1111/pbi.12078

Brader G, Mikkelsen MD, Halkier BA and Tapio Palva E (2006). Altering glucosinolate profiles modulates disease resistance in plants. Plant $J$. 46: 758-767. http://dx.doi.org/10.1111/j.1365-313X.2006.02743.x

Chen Y, Yan X and Chen S (2011). Bioinformatic analysis of molecular network of glucosinolate biosynthesis. Comput. Biol. Chem. 35: 10-18. http://dx.doi.org/10.1016/j.compbiolchem.2010.12.002

Gao H, Song A, Zhu X, Chen F, et al. (2012). The heterologous expression in Arabidopsis of a chrysanthemum Cys2/ His2 zinc finger protein gene confers salinity and drought tolerance. Planta 235: 979-993. http://dx.doi.org/10.1007/ s00425-011-1558-X

Gigolashvili T, Berger B, Mock HP, Müller C, et al. (2007a). The transcription factor HIG1/MYB51 regulates indolic glucosinolate biosynthesis in Arabidopsis thaliana. Plant J. 50: 886-901. http://dx.doi.org/10.1111/j.1365313X.2007.03099.x

Gigolashvili T, Yatusevich R, Berger B, Müller C, et al. (2007b). The R2R3-MYB transcription factor HAG1/MYB28 is a regulator of methionine-derived glucosinolate biosynthesis in Arabidopsis thaliana. Plant J. 51: 247-261. http:// dx.doi.org/10.1111/j.1365-313X.2007.03133.x

Gigolashvili T, Engqvist M, Yatusevich R, Müller C, et al. (2008). HAG2/MYB76 and HAG3/MYB29 exert a specific and coordinated control on the regulation of aliphatic glucosinolate biosynthesis in Arabidopsis thaliana. New Phytol. 177: 627-642. http://dx.doi.org/10.1111/j.1469-8137.2007.02295.x

Gigolashvili T, Yatusevich R, Rollwitz I, Humphry M, et al. (2009). The plastidic bile acid transporter 5 is required for the biosynthesis of methionine-derived glucosinolates in Arabidopsis thaliana. Plant Cell 21: 1813-1829. $\underline{\text { http://dx.doi. }}$ org/10.1105/tpc. 109.066399

Grubb CD and Abel S (2006). Glucosinolate metabolism and its control. Trends Plant Sci. 11: 89-100. http://dx.doi. org/10.1016/j.tplants.2005.12.006

Hirai MY, Sugiyama K, Sawada Y, Tohge T, et al. (2007). Omics-based identification of Arabidopsis Myb transcription factors regulating aliphatic glucosinolate biosynthesis. Proc. Natl. Acad. Sci. USA 104: 6478-6483. http://dx.doi. org/10.1073/pnas.0611629104

Genetics and Molecular Research 15 (3): gmr.15038381 
Jun BK, Seo SG, Kim JS, Lee Y, et al. (2011). Molecular cloning and expression analysis of Bro-GS-elong and Bro-myro from Brassica oleracea. Genes Genomics 33: 299-305. http://dx.doi.org/10.1007/s13258-011-0031-3

Kang YH, Kirik V, Hulskamp M, Nam KH, et al. (2009). The MYB23 gene provides a positive feedback loop for cell fate specification in the Arabidopsis root epidermis. Plant Cell 21: 1080-1094. http://dx.doi.org/10.1105/tpc.108.063180

Kim YB, Li X, Kim SJ, Kim HH, et al. (2013). MYB transcription factors regulate glucosinolate biosynthesis in different organs of Chinese cabbage (Brassica rapa ssp. pekinensis). Molecules 18: 8682-8695. http://dx.doi.org/10.3390/ molecules 18078682

Kos M, Houshyani B, Achhami BB, Wietsma R, et al. (2012). Herbivore-mediated effects of glucosinolates on different natural enemies of a specialist aphid. J. Chem. Ecol. 38: 100-115. http://dx.doi.org/10.1007/s10886-012-0065-2

Li F, Hasegawa Y, Saito M, Shirasawa S, et al. (2011). Extensive chromosome homoeology among Brassiceae species were revealed by comparative genetic mapping with high-density EST-based SNP markers in radish (Raphanus sativus L.). DNA Res. 18: 401-411. http://dx.doi.org/10.1093/dnares/dsr027

Li Y, Sawada Y, Hirai A, Sato M, et al. (2013). Novel insights into the function of Arabidopsis R2R3-MYB transcription factors regulating aliphatic glucosinolate biosynthesis. Plant Cell Physiol. 54: 1335-1344. http://dx.doi.org/10.1093/ pcp/pct085

Liu L, Guo W, Zhu X and Zhang T (2003). Inheritance and fine mapping of fertility restoration for cytoplasmic male sterility in Gossypium hirsutum L. Theor. Appl. Genet. 106: 461-469.

Livak KJ and Schmittgen TD (2001). Analysis of relative gene expression data using real-time quantitative PCR and the $2^{-\Delta \Delta C t}$ method. Methods 25: 402-408. http://dx.doi.org/10.1006/meth.2001.1262

Malik MS, Riley MB, Norsworthy JK and Bridges WJ (2010). Variation of glucosinolates in wild radish (Raphanus raphanistrum) accessions. J. Agric. Food Chem. 58: 11626-11632. http://dx.doi.org/10.1021/jf102809b

Miao H, Wei J, Zhao Y, Yan H, et al. (2013). Glucose signalling positively regulates aliphatic glucosinolate biosynthesis. J. Exp. Bot. 64: 1097-1109. http://dx.doi.org/10.1093/jxb/ers399

Mikkelsen MD, Petersen BL, Glawischnig E, Jensen AB, et al. (2003). Modulation of CYP79 genes and glucosinolate profiles in Arabidopsis by defense signaling pathways. Plant Physiol. 131: 298-308. http://dx.doi.org/10.1104/ pp. 011015

Mithen R, Faulkner K, Magrath R, Rose P, et al. (2003). Development of isothiocyanate-enriched broccoli, and its enhanced ability to induce phase 2 detoxification enzymes in mammalian cells. Theor. Appl. Genet. 106: 727-734.

Pan Y, Xu YY, Zhu XW, Liu Z, et al. (2014). Molecular characterization and expression profiles of myrosinase gene (RsMyr2) in radish (Raphanus sativus L.). J. Integr. Agric. 13: 1877-1888. http://dx.doi.org/10.1016/S2095$\underline{3119(13) 60644-9}$

Schuster J, Knill T, Reichelt M, Gershenzon J, et al. (2006). Branched-chain aminotransferase4 is part of the chain elongation pathway in the biosynthesis of methionine-derived glucosinolates in Arabidopsis. Plant Cell 18: 26642679. http://dx.doi.org/10.1105/tpc. 105.039339

Shan H, Chen S, Jiang J, Chen F, et al. (2012). Heterologous expression of the chrysanthemum R2R3-MYB transcription factor CmMYB2 enhances drought and salinity tolerance, increases hypersensitivity to ABA and delays flowering in Arabidopsis thaliana. Mol. Biotechnol. 51: 160-173. http://dx.doi.org/10.1007/s12033-011-9451-1

Shinozaki K and Yamaguchi-Shinozaki K (2007). Gene networks involved in drought stress response and tolerance. $J$. Exp. Bot. 58: 221-227. http://dx.doi.org/10.1093/jxb/erl164

Sønderby IE, Burow M, Rowe HC, Kliebenstein DJ, et al. (2010). A complex interplay of three R2R3 MYB transcription factors determines the profile of aliphatic glucosinolates in Arabidopsis. Plant Physiol. 153: 348-363. http://dx.doi. org $/ 10.1104 / \mathrm{pp} .109 .149286$

Stracke R, Holtgräwe D, Schneider J, Pucker B, et al. (2014). Genome-wide identification and characterisation of R2R3MYB genes in sugar beet (Beta vulgaris). BMC Plant Biol. 14: 249. http://dx.doi.org/10.1186/s12870-014-0249-8

Tamura K, Dudley J, Nei M and Kumar S (2007). MEGA4: molecular evolutionary genetics analysis (MEGA) software version 4.0. Mol. Biol. Evol. 24: 1596-1599. http://dx.doi.org/10.1093/molbev/msm092

Verdier J, Zhao J, Torres-Jerez I, Ge S, et al. (2012). MtPAR MYB transcription factor acts as an switch for proanthocyanidin biosynthesis in Medicago truncatula. Proc. Natl. Acad. Sci. USA 109: 1766-1771. http://dx.doi. org/10.1073/pnas.1120916109

Wang Y, Pan Y, Liu Z, Zhu X, et al. (2013). De novo transcriptome sequencing of radish (Raphanus sativus L.) and analysis of major genes involved in glucosinolate metabolism. BMC Genomics 14: 836. http://dx.doi.org/10.1186/1471-2164$\underline{14-836}$

Wilkins O, Nahal H, Foong J, Provart NJ, et al. (2009). Expansion and diversification of the Populus R2R3-MYB family of transcription factors. Plant Physiol. 149: 981-993. http://dx.doi.org/10.1104/pp.108.132795

Xie Z, Lee E, Lucas JR, Morohashi K, et al. (2010). Regulation of cell proliferation in the stomatal lineage by the

Genetics and Molecular Research 15 (3): gmr.15038381 
Arabidopsis MYB FOUR LIPS via direct targeting of core cell cycle genes. Plant Cell 22: 2306-2321. http://dx.doi. org/10.1105/tpc. 110.074609

Xu Y, Zhu X, Gong Y, Xu L, et al. (2012). Evaluation of reference genes for gene expression studies in radish (Raphanus sativus L.) using quantitative real-time PCR. Biochem. Biophys. Res. Commun. 424: 398-403. http://dx.doi. org/10.1016/j.bbrc.2012.06.119

Yang A, Dai X and Zhang WH (2012). A R2R3-type MYB gene, OsMYB2, is involved in salt, cold, and dehydration tolerance in rice. J. Exp. Bot. 63: 2541-2556. http://dx.doi.org/10.1093/jxb/err431

Zang Y, Zheng W, He Y, Hong S, et al. (2015). Global analysis of transcriptional response of Chinese cabbage to methyl jasmonate reveals JA signaling on enhancement of secondary metabolism pathways. Sci. Hortic. 189: 159-167. http://dx.doi.org/10.1016/j.scienta.2015.04.008

Zou Z, Ishida M, Li F, Kakizaki T, et al. (2013). QTL analysis using SNP markers developed by next-generation sequencing for identification of candidate genes controlling 4-methylthio-3-butenyl glucosinolate contents in roots of radish, Raphanus sativus L. PLoS One 8: e53541. http://dx.doi.org/10.1371/journal.pone.0053541

\section{Supplementary material}

Table S1. Protein information from NCBI used for construction of the phylogenetic tree.

Genetics and Molecular Research 15 (3): gmr.15038381 